\title{
Erratum: Genomic signatures of human and animal disease in the zoonotic pathogen Streptococcus suis
}

Lucy A. Weinert, Roy R. Chaudhuri, Jinhong Wang, Sarah E. Peters, Jukka Corander, Thibaut Jombart, Abiyad Baig, Kate J. Howell, Minna Vehkala, Niko Välimäki, David Harris, Tran Thi Bich Chieu, Nguyen Van Vinh Chau, James Campbell, Constance Schultsz, Julian Parkhill, Stephen D. Bentley, Paul R. Langford, Andrew N. Rycroft, Brendan W. Wren, Jeremy Farrar, Stephen Baker, Ngo Thi Hoa, Matthew T.G. Holden, Alexander W. Tucker, Duncan J. Maskell \& BRaDP1T Consortium

Nature Communications 6:6740 doi: 10.1038/ncomms7740 (2015); Published 31 Mar 2015; Updated 12 May 2015

This Article was originally published without the accompanying Supplementary Data 1-5. These files are now available in the HTML version of the Article; the PDF was correct from the time of publication. 